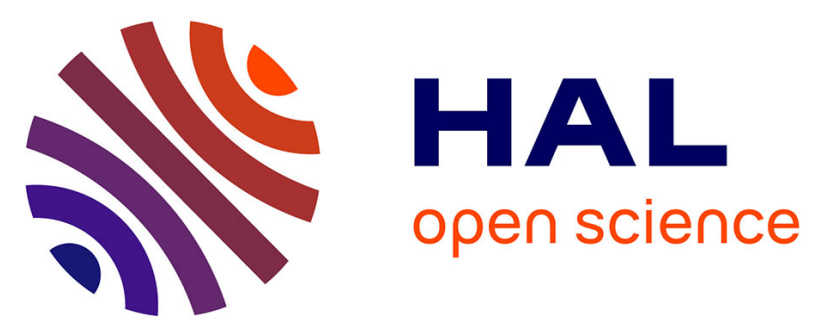

\title{
Mathematical modeling for the activation of t-lymphocytes: population balance modeling with non conventional growth law
}

Qasim Ali, Eric Touboul, Frédéric Gruy, Claude Lambert

\section{To cite this version:}

Qasim Ali, Eric Touboul, Frédéric Gruy, Claude Lambert. Mathematical modeling for the activation of t-lymphocytes: population balance modeling with non conventional growth law. 5th Asian Particle Technology Symposium, Jul 2012, Singapour, Singapore. pp.ISBN = 978-981-07-2518-1, 10.3850/978981-07-2518-1_195. hal-00721905

\section{HAL Id: hal-00721905 https://hal.science/hal-00721905}

Submitted on 31 Jul 2012

HAL is a multi-disciplinary open access archive for the deposit and dissemination of scientific research documents, whether they are published or not. The documents may come from teaching and research institutions in France or abroad, or from public or private research centers.
L'archive ouverte pluridisciplinaire HAL, est destinée au dépôt et à la diffusion de documents scientifiques de niveau recherche, publiés ou non, émanant des établissements d'enseignement et de recherche français ou étrangers, des laboratoires publics ou privés. 


\title{
Mathematical modeling for the activation of T-lymphocytes: Population balance modeling with non conventional growth law
}

\author{
Ali Qasim ${ }^{a^{*}}$, Touboul Eric ${ }^{a}$, Gruy Frederic ${ }^{a}$ and Lambert Claude ${ }^{b}$ \\ ${ }^{a}$ Ecole Nationale des Mines de Saint Etienne, France 42023 \\ ${ }^{\mathrm{b}}$ Immunology laboratory, University Hospital of Saint-Etienne, France \\ *Corresponding Author's E-mail: $\underline{\text { ali@emse.fr }}$
}

Keywords: T - lymphocytes, Cell Population Balances, Activation Kinetics, Hyperbolic Problems.

\begin{abstract}
This article focuses on the mathematical modeling techniques applied to the particulate processes in biological sciences particularly Immunology. T-cell activation is studied with respect to their surface protein (CD3) concentration that is described in-terms of two particulate processes, growth and birth processes. The production of new proteins and change in their concentrations (on cells) has created the idea of balance law in the form of hyperbolic problems. In order to add a new dimension to the problem, CPBM (Cell Population Balance Model) is used with respect to multi-proteins concentrations. A mathematical model is used to find analytical solution while Finite volume scheme is used to approximate numerically the solution of PBE (Population Balance Equation) in order to anticipate and analyze the density of activated T-cells according to the given initial protein concentration.
\end{abstract}

\section{Introduction}

Infections caused by viruses have various aspects depending upon the affection of organs in the body but the immunity against them is always provided by T-cells. The invasion is first detected by APCs (Antigen Presenting Cells) that absorb and cut viruses into small pieces (peptides) and locate them on their membrane. T-cells then recognize the pathogens that are displayed as peptides on the surface of APCs. The peptides are carried out by a membrane protein named MHC (Major Histo-compatibility Complex). First TCR (T-cell Receptor) combines with MHC along with a T-cell protein named CD3 [Carreno et al. 2006, Madrenas et al.1999, Margulies et al. 1997]. After recognition of the peptide, CD3-TCR proteins go inside the cell (internalization) that causes a decrease of CD3 protein on the surface of T-cell.

A sufficient internalization rate is mandatory to reach the threshold level for the full activation of Tcell [Rabinowitz et al.1996, Kalergis et al.2001, Williams et al. 1999]. In the process of activation, Tcells produce many proteins inside and on the surface that helps in preparing, informing and proliferating the required T-cells. As much T-cells will be produced by proliferation due to activation, as much viruses will be eliminated.

Cell population densities corresponding to various proteins can be measured by flow cytometry. So, the population density for the total CD3 can be experimentally determined. It has to be emphasized that this measurement is not available for each type of CD3 that is described below.

Several models are already computed and compared with published data with well defined peptides [Grakoui et al. 1999, Bidot et al. 2008, Sousa et al. 2000].

In this study, we will focus on the CD3 protein that has shown an interesting phenomenon in the Tcell: experimentally, it is observed that the $\mathrm{CD} 3$ protein concentration decreases during the activation process and then starts increasing by reappearing on the surface of T-cell probably in another place on the membrane. In short, we will be concerned about the case of $\mathrm{CD} 3$ ( surface protein) internalization as $\mathrm{CD} 3 \mathrm{i}$ (volume protein) and reappearance as $\mathrm{CD} 3 *$ (surface protein). 


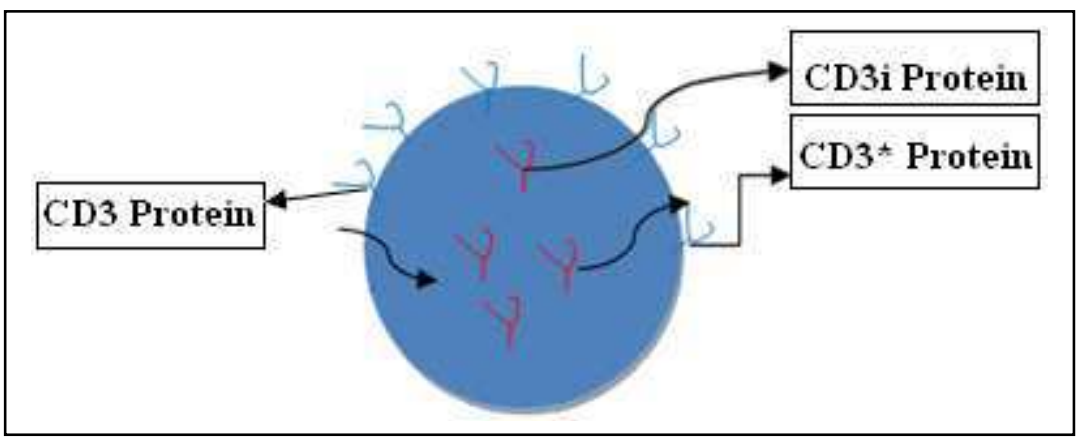

Figure 1: Membrane receptors reconverted to CD3

At a given time the T-cells have different concentrations of proteins depending on their initial protein (e.g. CD3) content and their collision instant with APC. The continuous variation in the population of activated T-cells needs the idea of balance law in cell population analysis. This leads to the use of Population Balance Equation (PBE) with the protein concentration as the internal coordinate. Population balance modeling is widely used in chemical engineering, pioneered by Hulburt and Katz [Hulburt et al.1964], and became popular in biological sciences since a decade. Up to now, some investigators used a PBE's system for several types of cells while the other ones only considered the dynamics of numerous proteins in a single T-cell. However, very few works have been dedicated to the coupling between PBE and complex kinetics in the T-cell. PBM efficiently describes the change in the population of individuals and their properties within a given interval of time. Changes are due to the appearance of newly activated T-cells and the activation process in each T-cell. In this study we will focus on the dynamics of the population of T-cells and its properties like CD3 protein concentration. Non monotonic concentration change as for CD3 leads to non trivial behavior of the PBE from a mathematical point of view. We propose in this paper a study of such a partial differential equation.

The second section of the paper presents the mathematical model. The third section is devoted to the derivation of the PBE. The fourth section compares analytical and numerical methods.

\section{Mathematical Model}

For each cell, the surface $\mathrm{CD} 3$ and $\mathrm{CD} 3^{*}$ concentrations are respectively noted $c_{1}(t)$ and $c_{2}(t)$, while the inner $\mathrm{CD} 3_{\mathrm{i}}$ concentration is noted $c_{i}(t)$. The total protein surface concentration, that can be measured by cytometry, is $c(t)=c_{1}(t)+c_{2}(t)$. For each cell, this concentration evolves, from the instant the cell get activated, in a way governed by kinetics laws.

The objective of the mathematical model developed here is to describe and predict, among the whole population of T-cell, the density $n(c, t)$ of T-cells having a total protein surface concentration $c$ at time $t$. Classically, $n(c, t)$ is solution of an hyperbolic population balance equation. The difficulty here is the non-monotony of the function $c(t)$, involves crossing between the characteristic curves and the opposite fluxes of populations.

The description of the mathematical model thus will be divided into two levels:

- The cell level describes the evolution of the surface protein concentration for each cell.

- The population level integrates the individual behavior into a population behavior to allow the computation of $n(c, t)$.

\section{2-1: Cell level}

For each individual T-Cell, the following steps can describe the beginning of the activation process [El-hentati et al. 2010], and the evolution of the protein concentration:

- Recognition of p-MHC and peptide by T-cell receptor after a collision event: $L+T \rightarrow L T$ (the parameters L and T represent APC and TCR respectively). This step will be called "activation". From this collision, the T-cell becomes active, and kinetic laws will describe the evolution of proteins concentrations. 
- CD3-TCR internalization: $\quad T \rightarrow T_{i}$

- Re-appearance of the irreversible CD3 protein $\left(\mathrm{CD} 3^{*}\right)$ on the surface $\quad T_{i} \rightarrow T^{*}$

For a given cell, activated at time 0 , a linear kinetic law gives the following equations:

$$
\frac{d c_{1}}{d t}=-k_{1} c_{1}, \quad \frac{d c_{i}}{d t}=k_{1} c_{1}, \quad \frac{d c_{2}}{d t}=k_{2} c_{i}
$$

with initial conditions : $c_{1}(0)=c_{10}, \quad c_{i}(0)=c_{2}(0)=0$

By integration: $c_{1}=c_{10} \exp \left(-k_{1} t\right), \quad c_{i}=c_{10}-c_{1}=c_{10}\left(1-\exp \left(-k_{1} t\right)\right)$

$$
\frac{d c_{2}}{d t}=k_{2} c_{10}-k_{2} c_{10} \exp \left(-k_{1} t\right), \quad c_{2}=k_{2} c_{10} t+\frac{k_{2}}{k_{1}} c_{10} \exp \left(-k_{1} t\right)-\frac{k_{2}}{k_{1}} c_{10}
$$

The expression of $c(t)$ is thus:

$$
c_{1}(t)+c_{2}(t)=c(t)=\left(1+\frac{k_{2}}{k_{1}}\right) c_{10} \exp \left(-k_{1} t\right)+k_{2} c_{10} t-\frac{k_{2}}{k_{1}} c_{10}=\alpha \exp \left(-k_{1} t\right)+\beta t+\gamma
$$

For a cell activated at time $\tau<t$, the expression of $\mathrm{c}$ has to be shifted. We can thus define a family of non monotonic curves indexed by $\tau$, noted $\tilde{c}(t, \tau)$, derived by translation from $\mathrm{c}(\mathrm{t})$ :

$$
\left\{\begin{array}{l}
c_{\tau}(t)=c(t-\tau)=a \exp \left(-k_{1}(t-\tau)+b(t-\tau)+d \text { if } t>\tau\right. \\
c_{\tau}(t)=0 \text { if } t<\tau
\end{array}\right.
$$

The following curves are generated for $\mathrm{k}_{1}=\mathrm{k}_{2}=2, \mathrm{c}_{10}=1$, with different activation times

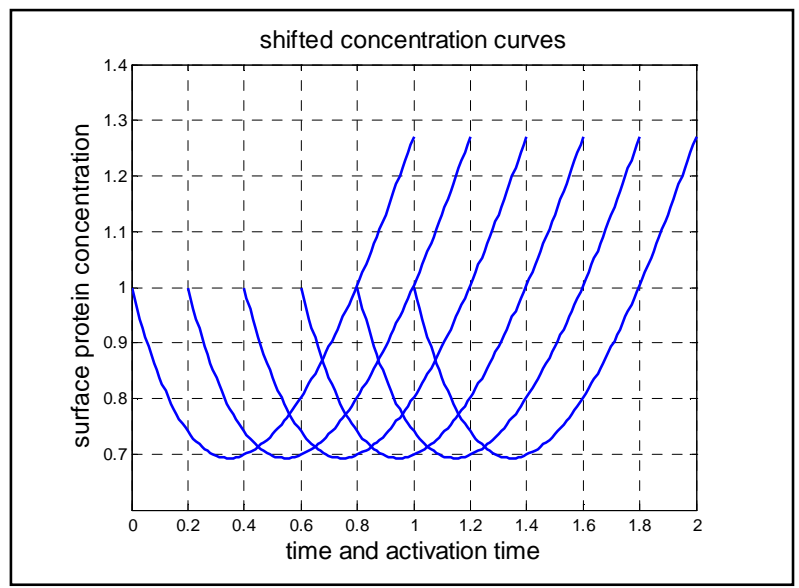

Figure 2: Surface concentration for individual cells

The curves are simply shifted because neither the coefficients $k_{i}$ nor the initial concentration $c_{10}$ depend on time. But one could imagine more complex situations.

The common minimum value for the curves is noted $\mathrm{c}_{\min }$. For $\tau=0, \mathrm{c}(\mathrm{t})=\mathrm{c}_{\min }$ occurs at a time noted $t^{*}$, the first time from which the different curves begins to cross each others. Realistically, the concentration of protein will be steady after some time due to finite space present on the cell surface. Here we are not considering the steady part of the problem otherwise there will be a change in the concentration of $c_{2}(t)$ as $\mathrm{dc}_{2} / \mathrm{dt}=\mathrm{k}_{2} \mathrm{c}_{\mathrm{i}}\left(\mathrm{c}_{10}-\mathrm{c}_{2}\right)$. This shows that the concentration is bounded for large values of time.

\section{2-2: Population level}

The activation is described by an activation rate function $b(\tau)$, number of cells activated per unit of time at time $\tau$. Classically, the rate of variation of the population of non activated cells is taken 
proportional to this population: $\frac{d N_{n a}}{d t}=-a N_{n a}$, where $N_{n a}=N_{0} \exp (-a t)$ is the density of non activated cells. The number of cell being activated at each time is taken proportional to the number of non activated cells, thus usually $b(t)$ is a decreasing exponential function.

Thus we will consider $b(\tau)=b_{0} \exp (-a \tau)$.

In the case when the curves $c_{\tau}(t)$ are monotonic, without crossing (this is the case here for $t<t^{*}$ ): For a given couple $(c, t)$ with $c>c_{\min }$, there is only one curve $c_{\tau}(t)$ passing through this point. Thus there is one corresponding $\tau(c, t)$ and one derivative $\frac{d c_{\tau}}{d t}(t)=\frac{d c}{d t}(t-\tau)$. Note that, the case where the curves are deduced by translation, $\frac{d c_{\tau}}{d t}$ can also be considered as a function of $c$ only. $\frac{d c_{\tau}}{d t}(c)=\frac{d c}{d t}(c)$. During a time step $d t$, the number of cells activated between $\tau$ and $\tau+d \tau$ is travelling along the characteristic curve $c(t, \tau)$, and the conservation of cells gives: $n(c, t)|d c|=b(\tau(c, t)) d t$. The population density $n(c, t)$, is thus simply linked to the slope of this curve and to the activation rate $b(\tau)$ :

$$
n(c, t)=\frac{b(\tau(c, t))}{\left|\frac{d c}{d t}(t-\tau(c, t))\right|}
$$

This gives a simple way, almost analytical, to derive $n$ from $b$ and the slopes $\frac{d c}{d t}$. Here, $\tau(c, t)$ should be determined numerically. One can observe that $n(c, t)$ tends to infinity when $c$ tends to $\mathrm{c}_{\min }$, since the derivative $\frac{d c}{d t}\left(c=c_{\text {min }}\right)=0$. But the integral of the function $n(c, t)$ remains finite, and thus the number of cells in any interval $\left[c_{\min }, c\right]$ can be computed. Moreover, $\int_{c_{\min }}^{c} n(c, t) d c \rightarrow 0$ when $c \rightarrow c_{\min }$. Numerically, more often, the value of $n(c, t)$ at a node represent its integral on a cell divided by the size of the cell. Thus the numerical values of $n(c, t)$ at $c=c_{\min }$ for a given mesh will be finite. Nevertheless this behaviour may affect the numerical accuracy.
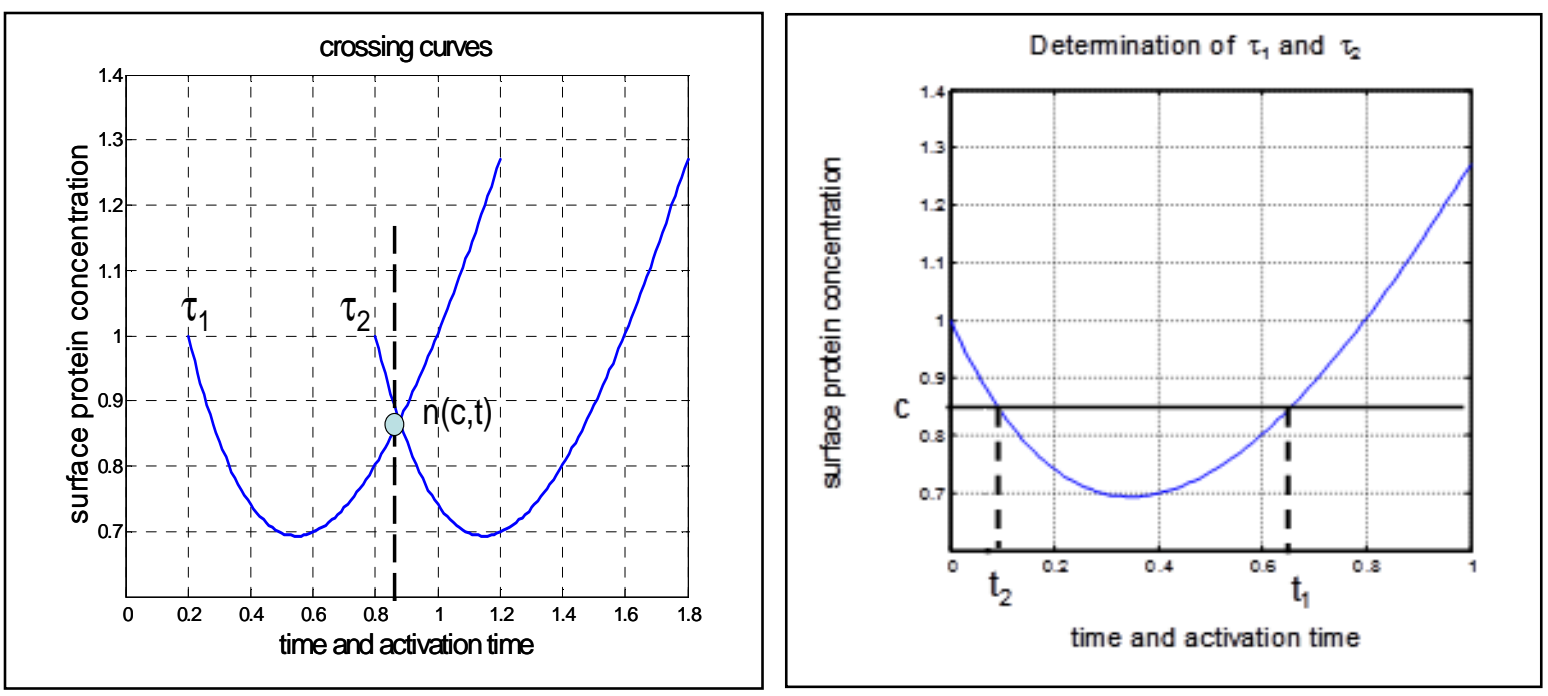

Figure 3: Numerical computation of $\tau_{1}$ and $\tau_{2}$ 
In the case where $t>t^{*}$, for a given couple $(c, t)$ with $c>c_{\min }$, there are two curves passing through the point, i.e. $\tau_{1}<\tau_{2}$, with their corresponding derivatives. The value of $\tau$ has been obtained either numerically, with a Newton-Raphson method, either by approximation of $c(t)$ by a polynomial and then the determination of the roots of this polynomial.

$t_{1}(c)<t_{2}(c)$ are obtained from the curve $c(t)$, then the $t$ values are deduced : $\tau_{i}(c, t)=t-t_{i}(c)$. The derivatives are computed from the expression of $c$ versus $t$.

$$
n(c, t)=n_{1}(c, t)+n_{2}(c, t)=\frac{b\left(\tau_{1}(c, t)\right)}{\left|\frac{d c}{d t}\left(t-\tau_{1}(c, t)\right)\right|}+\frac{b\left(\tau_{2}(c, t)\right)}{\left|\frac{d c}{d t}\left(t-\tau_{2}(c, t)\right)\right|},
$$

$n_{1}$ and $n_{2}$ correspond to the two populations activated at $\tau_{1}$ and $\tau_{2}$, having a protein concentration $c$ at time $t$. For the first population, the concentration is increasing, while for the second, it is decreasing.

The results obtained by this simple method are displayed on figure 4 . For this example, $k_{1}=k_{2}=2$, $c_{10}=1$, and $b(\tau)=\exp (-\tau)$ and the different times are: 0.1, 0.6, 1.1 and 1.6. The initial discontinuity propagates downward, and when $c_{\min }$ is reached, the discontinuity propagates upward. It is important to mention here that $n\left(c_{\min }, t\right)$ is not computed since it tends to infinity.

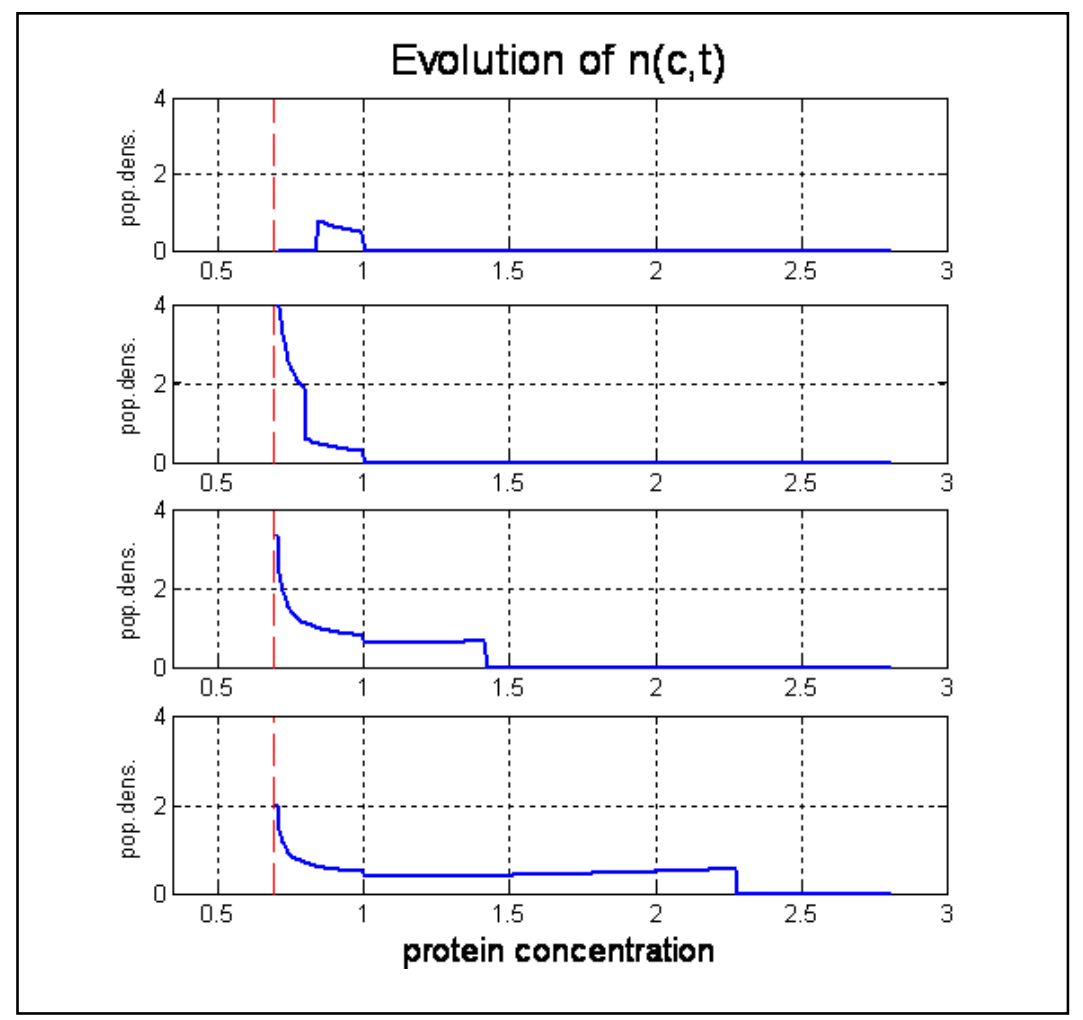

Figure 4: Analytical way of evolution of the surface protein density $n(c, t)$

\section{3: Derivation of a population balance equation}

The method presented above, rather simple, will not be suitable in more general cases, for example when the initial concentration $c_{10}$ is replaced by a distribution function. It is more convenient then to adapt the classical conservation law approach (hyperbolic equation) to this particular case of non monotonic concentration curve. 
Let us recall that, in case of monotonic function $c=c(t)$, the conservation equation can be written as a scalar transport equation, with the concentration $\mathrm{c}$ an inner variable (space variable in classical advection equation). For example, in our case, at a time $t<t^{*}$, the population flux at concentration $c$ is: $q=\frac{d c_{\tau(c, t)}}{d t}(c) \cdot n(c, t)$. The populations are travelling downward on the $c$-axis. Since the curves are deduced by translation in time, the derivative only depends on $c$. This derivative, classically called the growth function, is noted $G(c)=\frac{\partial c}{\partial t}(c)$.

Thus the conservation equation and the initial and boundary conditions can be written:

$$
\begin{cases}\frac{\partial n(c, t)}{\partial t}+\frac{\partial}{\partial c}(G(c) \cdot n(c, t))=0 & \begin{array}{l}
\text { We can also write the conservation equation on a non conservative } \\
n(c, 0)=0
\end{array} \\
n\left(c_{10}, t\right)=\frac{b(t)}{G\left(c_{10}\right)} & \frac{\partial n(c, t)}{\partial t}+G(c) \frac{\partial n(c, t)}{\partial c}=-G^{\prime}(c) n(c, t)\end{cases}
$$

Along a characteristic curve beginning at $\tau$, of equation: $\left\{\begin{array}{l}\frac{d c_{\tau}}{d t}=G(c) \\ c_{\tau}(0)=c_{10}\end{array}\right.$, the variation of $\mathrm{n}(\mathrm{c}(\mathrm{t}), \mathrm{t})$ is $\frac{d n(t)}{d t}=-n(t) G^{\prime}(c), \quad n(t)=n_{0} \exp \left(-\int_{0}^{t}\left(\frac{d G(c)}{d c}\right)(t) d t\right)$

For $t>t^{*}$, characteristics are crossing each others. At each point $(c, t)$, for $c>c_{\min }$, are present two opposite fluxes:

- the flux of cells activated at time $\tau_{1}$ whose concentration is increasing, and thus who travel upward on the $\mathrm{c}$ axis. If the density of population of these cells is noted $n_{1}$, their flux is equal to $q_{1}=G_{1}(c) \cdot n_{1}(c, t)$ with $G_{1}(c)=\frac{d c_{\tau_{1}(c, t)}}{d t}(c)$

- the flux of cells activated at time $\tau_{2}>\tau_{1}$, whose concentration is decreasing and travel downward on the c axis with a flux $q_{2}=G_{2}(c) \cdot n_{2}(c, t), G_{2}(c)=\frac{d c_{\tau_{2}(c, t)}}{d t}(c)$, where $n_{2}$ is the population density of these cells.

The resulting total flux can be computed: $G=\frac{n_{1} G_{1}(c)+n_{2} G_{2}(c)}{n_{1}+n_{2}}$, with $\mathrm{G}_{2}<0$. But it seems to be not possible to compute the "equivalent" growth rate $G$ as a function of c only, or of $\mathrm{n}$, since $n_{1}$ and $n_{2}$ need to be known.

A way of solving this problem is to write one conservation equation for each population, and then to compute the total density $n(c, t)$ as: $n=n_{1}+n_{2}$

$\frac{\partial n_{i}(c, t)}{\partial t}+\frac{\partial}{\partial c}\left(G_{i}(c) \cdot n_{i}(c, t)\right)=0, \quad i=1,2$

The boundary conditions for the first equation are: $\left\{\begin{array}{l}n_{1}\left(c_{1}, 0\right)=0 \\ n_{1}\left(c_{10}, t\right)=n_{0}\end{array}\right.$.

But for the second equation, the value of $\mathrm{n}$ at $c=c_{\text {min }}$ is not finite. A way to overpass this problem is to consider a little fixed interval $\left[c_{\min }, c_{\min }+d c\right]$ on which $n(c, t)$ is replaced by its mean value.

This is naturally the case in the numerical solving, using a finite volume method. On each cell, the unknown will be the mean value of $n$ on the cell. 


\section{4: Numerical resolution of the conservation equation}

We use here a simple one order upwind explicit finite volume scheme. $n_{i}^{k} \approx n\left(c_{i}, t^{k}\right)$ is the approximate average value of $n(c, t)$ at time $t^{k}$ on a block centred on $c_{i} . F_{i-1 / 2}^{k}$ and $F_{i+1 / 2}^{k}$ are the fluxes respectively upwind and downwind at the boundary of this block. The scheme is of the following form:

$$
\left(n_{i}^{k+1}-n_{i}^{k}\right) \Delta c+\left(F_{i+1 / 2}^{k}-F_{i-1 / 2}^{k}\right) \Delta t=0
$$

The fluxes are computed upwind on the following way: $\left\{\begin{array}{l}F_{i-1 / 2}^{k}=G\left(c_{i-1 / 2}\right) n_{i-1} \\ F_{i+1 / 2}^{k}=G\left(c_{i+1 / 2}\right) n_{i}\end{array}\right.$

The two fluxes are treated in the continuity on one to the other, the first block on the c-axis $\left(c=c_{\text {min }}\right)$ making the connexion. But each equation is solved on a separate grid, since one have to distinguish $n_{1}$ and $n_{2}$. Then, between $c_{\text {min }}$ and $c_{0}, n_{1}$ and $n_{2}$ are added to obtain the density $n(c, t)$. The principle of the scheme is exposed in fig 5:

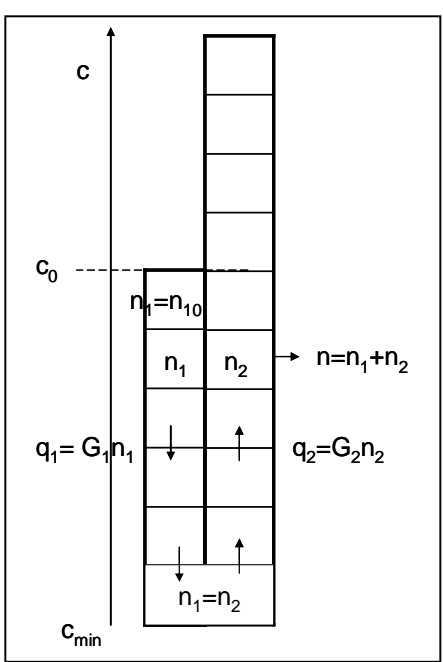

Figure 5: Principle of the numerical scheme

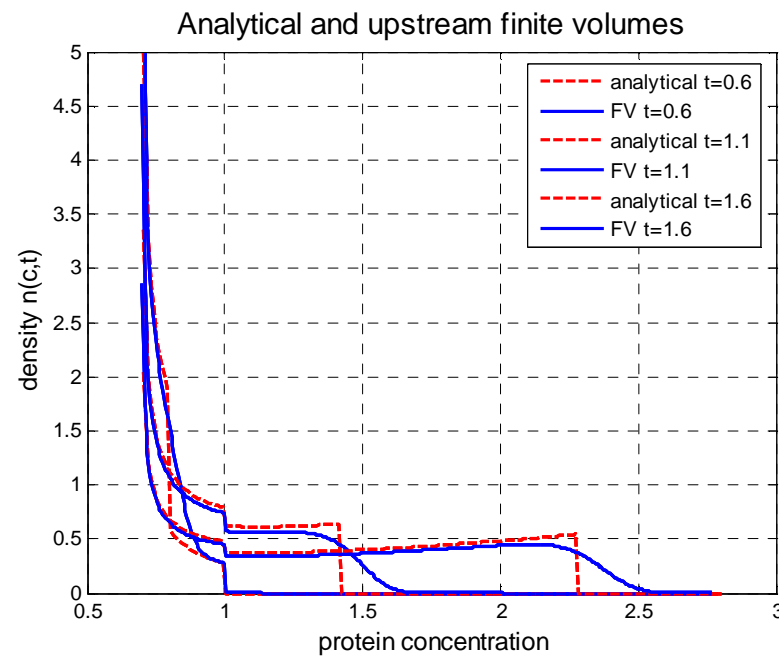

Figure 6: Results with the first order finite volume scheme

With such a scheme, known to be stable providing the classical CFL condition on the time step is respected, present the drawback of developing a high numerical diffusion. The comparison with the analytical solution, on the same data, is presented above in figure 6 . The time step is 0.0025 while the c-step was 0.005 .

These results present high numerical diffusion, a well known drawback of this scheme. The discontinuities are dumped. Nevertheless, the results are quite comparable to the analytical solution.

In order to decrease the numerical diffusion around the discontinuity front, we can use the following idea:

The velocity of the density discontinuity on the c axis can be numerically derived from the computation of the growth rates $\mathrm{G}(\mathrm{c})$. This velocity is computed at each boundary between blocks in the finite volume scheme. Thus, the time at which the propagating discontinuity reaches a given block can be numerically approximated. In order to avoid numerical diffusion around the propagating discontinuity, the flux on the boundary between two grid blocks can be set to 0 until this boundary is reached by the front computed from G(c) (diffusion limitation scheme). But this method does not prevent diffusion behind the front.

The result derived is then very close to the analytical solution, as shown in fig 7 . 
Analytical and upstream finite volumes with difusion limitation

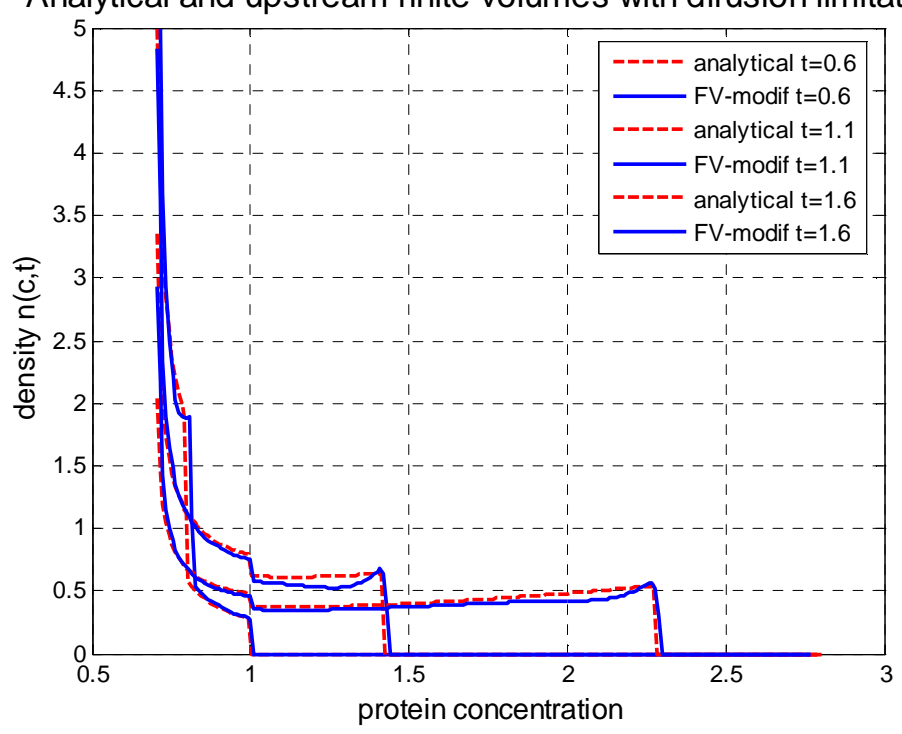

Figure 7: results with finite volume scheme and diffusion limitation

\section{5: Acknowledgement}

We are highly thankful to EMSE (Ecole des Mines de Saint Etienne) for their financial and moral support.

\section{6: Conclusion}

We have developed a model for CD3 protein evolution at the surface of a population of T-cells. The resulting population balance equation shows, in the studied case, two difficulties versus what is commonly encountered:

- Despite the equation is linear, the characteristics curves are non monotonic, they cross each others, involving opposite fluxes for two parts of the population. Therefore, the way the population balance equation is usually written, i.e. for the whole population, seems no more valid.

- The flux becomes null at one point of the concentration axis, involving an infinite value for the population density.

The objective of this study is well established by proposing an analytical solution based on characteristic curves analysis, as well as a numerical finite volume scheme that may allow us to go further in the complexity of the problem treated.

Our future work will be directed towards improvement of the finite volume scheme to better decrease the numerical diffusion and accurately represent the discontinuities fronts, and also towards the treatment of a more complex and realistic problem, where the initial concentration of $\mathrm{CD} 3$ on the Tcell is no more uniform but follows a distribution law, and where the characteristics can be of more complex shapes.

The second order accurate Lax-Wendroff difference scheme can be used to alleviate the numerical diffusion but they give oscillatory solutions near the discontinuities. On the other hand upwind difference schemes give a smooth curve by monotonically varying in the region even though their accuracy is not so good. It is intimated that the high resolution schemes can be used using the notion of flux limiter and Total Variation Diminishing (TVD). The MUSCL finite volume scheme [Sohn et al. 2005] can also give better accuracy with discontinuities of such type. The idea of implicit methods is given in the literature that gives $2^{\text {nd }}$ order accuracy like Crank-Nicolson Method.

From the literature review it is observed that the problem of oscillation in the solution due to discontinuities is the main cause of diffusion that can be reduced by using any flux limiter second order method. 


\section{References}

1. Carreno L. J., Gonzalez P. A., Kalergis A. M. Modulation of T cell function by TCR/pMHC binding kinetics, Immunobiology, 211, 47-64, 2006.

2. Madrenas J. Differential signalling by variant ligands of the $T$ cell receptor and the kinetic model of T cell activation, Life Sci, 64, 717-731, 1999.

3. Margulies D. H. Interactions of TCRs with MHC-peptide complexes: a quantitative basis for mechanistic models, Curr Opin Immunol, 9, 390-395, 1997.

4. Rabinowitz J. D., Beeson C., Lyons D. S., Davis M. M., McConnell H. M. Kinetic discrimination in T-cell activation. Proc Natl Acad Sci USA, 93, 1401-1405, 1996.

5. Kalergis A. M., Boucheron N., Doucey M. A., Palmieri E., Goyarts E. C., Vegh Z., Luescher I. F. and Nathenson S. G. Efficient T cell activation requires an optimal dwell-time of interaction between the TCR and the pMHC complex, Nat Immunol, 2, 229-234, 2001.

6. Williams C. B., Engle D. L., Kersh G. J., Michael White J. and Allen P. M. A kinetic threshold between negative and positive selection based on the longevity of the $\mathrm{T}$ cell receptor-ligand complex, J Exp Med, 189, 1531-1544, 1999.

7. Grakoui A., Bromley S. K., Sumen C., Davis M. M., Shaw A. S., Allen P. M. and Dustin M. L. The immunological synapse: a molecular machine controlling $\mathrm{T}$ cell activation, Science, 285, 221-227, 1999.

8. Bidot C., Gruy F., Haudin C. S., El Hentati F., Guy B. and Lambert C. Mathematical modeling of T-cell activation kinetic, J Comput Biol, 15, 105-128, 2008.

9. Sousa J. and Carneiro J. A mathematical analysis of TCR serial triggering and downregulation, Eur J Immunol, 30, 3219-3227, 2000.

10. Hulburt, H. M., Katz, S. Some problems in particle technology, Chem Eng Sci, 19, 555-574, 1964.

11. El-Hentati F.Z., Gruy F., Iobagiu C., Lambert C. Variability of CD3 membrane expression and T cell activation capacity, Clinical Cytometry, 78B, 105-114, 2010.

12. Sohn S. A new TVD-MUSCL scheme for hyperbolic conservation laws, Computers \& Mathematics with Applications, 50, 231-248, 2005. 